\title{
NOUVELLE
}

\section{Sphingomyélinases et ostéogenèse imparfaite}

Isabelle Aubin, Jean-Louis Guénet

Le syndrome d'ostéogenèse imparfaite regroupe un ensemble de maladies d'origine génétique qui affectent principalement les os, mais aussi les dents et parfois la peau. Le syndrome en question affecte environ un nouveau-né pour 15000 naissances et se classe donc parmi les maladies «orphelines». La nature et la sévérité des symptômes sont très variables, mais une caractéristique commune à tous les patients est une grande fragilité osseuse qui se traduit par des fractures «spontanées » et dont découle l'appellation de «maladie des os de verre ». Une première classification établie en 1979 et reflétant la diversité du spectre clinique recensait quatre grands types d'ostéogenèse imparfaite (I à VI) tous liés à des mutations affectant les gènes codant pour les chaînes $\alpha 1$ ou $\alpha 2$ du procollagène de type I (COLIAl et COL1A2) [1]. La classification en question a été révisée récemment et étendue à trois nouveaux types ( $\mathrm{V}$ à VII) qui regroupent $10 \%$ à $15 \%$ des cas [2]. Pour ces trois derniers types, aucune mutation n'a été identifiée dans les gènes du collagène.

De nombreux modèles animaux d'ostéogenèse imparfaite, avec un défaut du collagène, ont été décrits et ont permis d'améliorer notre connaissance de la physiopathologie de ces maladies chez l'homme. La mutation récessive fragilitas ossium (symbole fro) (Figure 1), découverte à I'Institut Pasteur (Paris, France) il y a plusieurs années, est le seul modèle d'ostéogenèse imparfaite non collagène-dépendante. Cette particularité fait de cette souris un modèle intéressant pour la recherche de gènes encore inconnus mais susceptibles d'être impliqués dans l'ostéogenèse. Le clonage de la mutation fro constitue aussi un point de départ pour l'identification de gènes candidats impliqués chez des patients atteints d'ostéogenèse imparfaite non collagène-dépendante.

Les premières études cliniques histologiques et radiographiques effectuées sur les mutants fro/fro ont mis en évidence un défaut de minéralisation, d'importantes déformations du squelette, un rachitisme, ainsi qu'une relative fragilité des homozygotes pendant les premiers jours de la vie [3] (Figure 2). Ces observations initiales ont été largement confirmées et validées par les travaux de Muriel et al., qui ont écarté l'hypothèse d'un défaut du collagène de type I [4], puis par ceux de Sillence et al. [5]. Plus récemment, en collaboration avec l'équipe de Michel Goldberg de la Faculté de chirurgie dentaire de Montrouge (France), nous avons décrit, chez les mutants, un phénotype de dentinogenèse imparfaite, un symptôme que l'on retrouve chez de nombreux patients atteint d'ostéogenèse imparfaite.

Par une approche de clonage positionnel, nous avons localisé la mutation fro sur le chromosome 8 dans un intervalle de $980 \mathrm{~kb}$ contenant 26 gènes. Compte tenu de leur profil d'expression et des données de la littérature, 10 de ces gènes ont été retenus comme des candidats de premier ordre. Le profil d'expression analysé chez des souris de 3 jours, homozygotes (fro/fro) et sauvages $(+/+)$, en amplifiant par RTPCR les exons terminaux des ADNc des 10 gènes candidats, nous a orienté sur le gène Smpd3 codant pour la sphingomyélinase neutre 2 (nSMase2). L'ana-
Département de Biologie du développement, Institut Pasteur, 25, rue du Docteur Roux, 75724 Paris Cedex 15, France. guenet@pasteur.fr

lyse de la séquence génomique de l'allèle Smpd3 chez les mutants a révélé une délétion de $1758 \mathrm{pb}$, impliquant la fin de l'intron 8 et le site accepteur d'épissage, la totalité de la séquence codante de l'exon 9, ainsi qu'une partie du 3' UTR. Le séquençage du transcrit mutant nous a permis de vérifier que la partie non délétée de l'intron 8 était transcrite chez les souris fro/fro. Cette séquence s'achève par un codon stop en amont de la délétion, ce qui suggère que, lors de sa traduction, 13 acides aminés d'origine intronique se substituent chez les mutants aux 33 acides aminés terminaux de la protéine normale. Ce remaniement entraîne la perte d'une histidine nécessaire à l'activité catalytique de la protéine et aboutit à la perte de l'activité enzymatique chez les souris mutantes. Cette hypothèse a été confirmée par le laboratoire de Robert Salvayre (Inserm U.466, Toulouse, France), par la démonstration d'une activité quasi inexistante de la nSMase2 chez les souris fro/fro et comparable à celle mesurée chez des souris

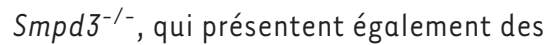
malformations du squelette [6].

Les sphingomyélinases (SMases), également appelées sphingomyéline-phosphodiestérases (SMPD), interviennent dans le catabolisme de la sphingomyéline, l'un des principaux constituants des bicouches lipidiques des membranes. II s'agit d'enzymes ubiquitaires qui hydrolysent la sphingomyéline en phosphocholine et céramide. Les céramides sont ensuite hydrolysés par une céramidase en sphingosine, laquelle est, à son tour, phosphorylée par une kinase pour donner la sphingosine-1-phosphate, ou SIP. 


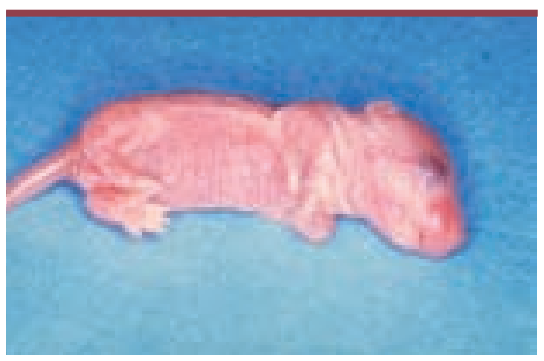

Figure 1. Souriceau nouveau-né fro/fro. La déformation des membres, notamment antérieurs, est très évidente dès la naissance. Le phénotype s'améliorera avec l'âge.

Les enzymes et métabolites de la voie sphingomyéline/céramide interviennent dans de nombreux mécanismes de signalisation, ainsi que dans la régulation de processus cellulaires majeurs tels que la prolifération cellulaire, l'apoptose ou encore la cytodifférenciation.
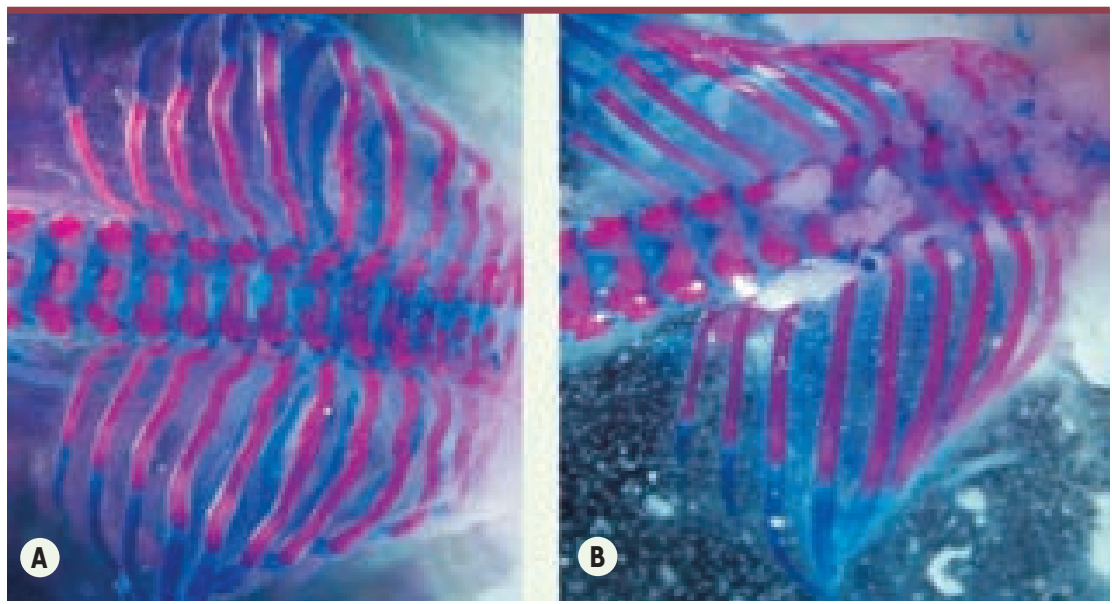

Figure 2. Colorations de squelettes thoraciques au rouge d'alizarine/bleu alcian. A. Photographie présentant une vue dorsale d'une souris de phénotype mutant âgée d'un jour. On peut noter une importante malformation des côtes ainsi que de nombreuses fractures. B. Photographie présentant une vue dorsale d'une souris de phénotype sauvage du même âge. Les côtes présentent un aspect harmonieux et régulier. pas encore parfaitement décryptées, mais il est désormais évident que la sphingomyélinase neutre 2 est un facteur important de régulation du développement osseux. La souris fragilitas ossium en est un bon exemple. $\nabla$

Sphingomyelinases and osteogenesis imperfecta

\section{RÉFÉRENCES}

1. Sillence DO, Senn A, Danks DM. Genetic heterogeneity in osteogenesis imperfecta. J Med Genet 1979; 16: 101-16.

2. Rauch F, Glorieux FH Osteogenesis imperfecta. Lancet $2004 ; 363$ : 1377-85.

3. Guenet JL, Stanescu R, Maroteaux P, et al. Fragilitas ossium : a new autosomal recessive mutation in the mouse. J Hered $1981 ; 72: 440-1$

4. Muriel MP, Bonaventure J, Stanescu R, et al. Morphological and biochemical studies of a mouse mutant (fro/fro) with bone fragility. Bone1991; $12: 241-8$.

5. Sillence D0, Ritchie HE, Dibbayawan T, et al. Fragilitas ossium (fro/fro) in the mouse: a model for a recessively inherited type of osteogenesis imperfecta. Am J Med Genet 1993 ; 45 : 276-83.

6. Stoffel W, Jenke B, Block B, et al. Neutral sphingomyelinase $2(\operatorname{smpd} 3)$ in the control of postnatal growth and development. Proc Natl Acad Sci USA 2005 ; 102 : 4554-9.

7. Takeda H, Ozaki K, Yasuda $\mathrm{H}$, et al. Sphingomyelinase and ceramide inhibit formation of F-actin ring in and bone resorption by rabbit mature osteoclasts. FEBS Lett 1998 ; 422 : 255-8.

8. Goldberg M, Boskey AL. Lipids and biomineralizations. Prog Histochem Cytochem 1996; $31: 1-187$.

9. Wu LN, Genge BR, Kang MW, et al. Changes in phospholipids extractability and composition accompany mineralization of chicken growth plate cartilage matrix vesicles. J Biol Chem 2002; 277 : 5126-33.

10. Chae SS, Paik JH, Allende ML, et al. Regulation of limb development by the sphingosine l-phosphate receptor Slpl/EDG-1 occurs via the hypoxia/VEGF axis. Dev Biol $2004 ; 268$ : 441-7.

11. Grey A, Xu X, Hill B, et al. Osteoblastic cells express phospholipid receptors and phosphatases and proliferate in response to sphingosine-1-phosphate. Calcif Tissue Int $2004 ; 74$ : 542-50.

12. Hla T. Physiological and pathological actions of sphingosine 1-phosphate. Semin Cell Dev Biol 2004 ; $15: 513-20$. 